anales de psicología, 2012, vol. $28, \mathrm{n}^{\circ} 3$ (octubre), 720-727 http://dx.doi.org/10.6018/analesps.28.3.156011
(C) Copvright 2012: Servicio de Publicaciones de la Universidad de Murcia. Murcia (España) ISSN edición impresa: 0212-9728. ISSN edición web (http://revistas.um.es/analesps): 1695-2294

\title{
Psicopatología y funciones ejecutivas en adictos a la cocaína
}

\author{
Gloria García-Fernández ${ }^{1 *}$, Olaya García-Rodríguez ${ }^{1}$, Roberto Secades-Villa ${ }^{1}$, \\ Emilio Sánchez-Hervás ${ }^{2}$ y José Ramón Fernández-Hermida ${ }^{1}$ \\ ${ }^{1}$ Grupo de Conductas Adictivas. Departamento de Psicología. Universidad de Oviedo (España) \\ ${ }^{2}$ Unidad de Conductas Adictivas de Catarroja. Agencia Valenciana de Salud Pública. Dpto. 10. Valencia (España)
}

\begin{abstract}
Resumen: El objetivo de este estudio fue evaluar la presencia de alteraciones psicopatológicas en una muestra de adictos a la cocaína y examinar la relación existente entre estas alteraciones y el rendimiento neuropsicológico en tareas de funcionamiento ejecutivo. La muestra se compuso por 50 participantes que habían solicitado tratamiento por su adicción a la cocaína. En el inicio del tratamiento, se aplicó a cada sujeto el EuropASI, el SCL-90-R, el BDI y una batería neuropsicológica compuesta por varias tareas sensibles a la detección de alteraciones en diferentes componentes de las funciones ejecutivas. Los resultados mostraron que al menos un $26 \%$ de la muestra puntuaba por encima del punto de corte de riesgo psicopatológico establecido en cada una de las escalas del SCL-90-R y que el $81,6 \%$ presentaba síntomas depresivos. Un mayor nivel de depresión y de ansiedad fóbica se relacionaron con la presencia de dificultades atencionales y de velocidad de procesamiento de información. No se encontraron relaciones estadísticamente significativas entre el resto las dimensiones psicopatológicas evaluadas y la ejecución en las distintas tareas neuropsicológicas.

Palabras clave: Adicción a la cocaína; psicopatología; rendimiento neuropsicológico; funciones ejecutivas.
\end{abstract}

\section{Introducción}

La adicción a la cocaína es un fenómeno especialmente complejo modulado por factores de diversa índole y relacionado con complicaciones médicas, alteraciones neuropsicológicas y neuroanatómicas, malestar psicopatológico y dificultades laborales, legales y familiares (Terán, Casete, \& Climent, 2008).

Numerosos estudios han confirmado la presencia de alteraciones neuropsicológicas en la adicción a la cocaína, y aunque el perfil encontrado dista de ser consistente, el mayor tamaño del efecto y acuerdo se encuentra en la presencia de alteraciones en la atención y en las funciones ejecutivas (Jovanovski, Erb, \& Zakzanis, 2005; Lorea, FernándezMontalvo, Tirapu-Ustarroz, Landa, \& López-Goñi, 2010; Ramos-Cejudo \& Iruarrizaga Díez, 2009; Verdejo-García \& Bechara, 2009).

Durante los últimos años el estudio de las alteraciones neuropsicológicas en la adicción a la cocaína y a otras drogas ha ido avanzando y han surgido nuevas líneas de investigación de gran interés científico y clínico (Sociedad Española de Toxicomanías, 2011). Estas líneas de investigación se han centrado en el análisis de la relación entre las alteraciones en las funciones ejecutivas y la gravedad de la adicción (Verdejo, Orozco-Gimenez, Meersmans Sánchez-Jofre, Aguilar de Arcos, \& Pérez-García, 2004), los subcomponentes de las funciones ejecutivas preservados y afectados por el consumo de drogas (Fernández-Serrano, Pérez-García,

* Dirección para correspondencia [Correspondence address]: Gloria García-Fernández. Grupo de Conductas Adictivas. Departamento de Psicología. Universidad de Oviedo. Plaza Feijoo, s/n. 33003 Oviedo (España).E-mail: gloriagarciafdez@gmail.com

\begin{abstract}
Title: Psychopathology and executive functions in cocaine addicts. Abstract: The aim of this study was to assess the presence of psychopathological symptoms in a sample of cocaine addicts and to examine the relationship between these symptoms and neuropsychological performance on executive functioning tasks. Fifty treatment-seeking outpatients for cocaine addiction participated. The EuropASI, the SCL-90-R, the BDI and a neuropsychological protocol, comprising different tasks sensitive to the detection of alterations in different components of the executive functions, were collected for each subject at the beginning of treatment. Results showed that at least $26 \%$ of the sample scored above the psychopathological risk cutoff of the dimensions of the SCL-90-R and 81.6\% showed depressive symptoms at treatment entry assessment. Only a higher level of depression and phobic anxiety were associated with attentional difficulties and information processing speed. There were no significant associations between other psychopathological dimensions and neuropsychological test performance scores.

Key words: Cocaine addiction; psychopathology; neuropsychological per-
\end{abstract} formance; executive functions.

Schmidt Río-Valle, \& Verdejo-García, 2010; FernándezSerrano, Pérez-García, \& Verdejo-García, 2011), el curso temporal y evolutivo de estos déficits (Di Sclafani, TolouShams, Price, \& Fein, 2002; García Fernández, García Rodríguez, Secades Villa, Fernández Hermida, \& Sánchez Hervás, 2010; Schrimsher \& Parker, 2008) y la utilidad de la evaluación neuropsicológica de las funciones ejecutivas como factor pronóstico sobre el éxito de la terapia y como herramienta de elección y adaptación del tratamiento (Aharonovich et al., 2006; Pace-Schott et al., 2008; Severtson, von Thomsen, Hedden, \& Latimer, 2010).

Por otra parte, los sujetos adictos a la cocaína presentan sintomatología psicopatológica, asiduamente sintomatología depresiva y de ansiedad, que influyen en la eficacia de los programas de tratamiento (Herrero, Domingo-Salvany, Torrens, \& Brugal, 2008; López Durán \& Becoña Iglesias, 2006). Sin embargo, pocos estudios han explorado la relación entre rendimiento en tareas de funcionamiento ejecutivo y comorbilidad psicopatológica y, en la mayoría de los casos, no se ha encontrado una relación clara. Rosselli, Ardila, Lubomski, Murray, \& King (2001) evaluaron las alteraciones psicopatológicas y el rendimiento neuropsicológico de una muestra de 42 dependientes a la cocaína. Aunque se confirmó la presencia de alteraciones psicopatólogicas y neuropsicológicas en la muestra de participantes, no se encontró una relación significativa entre ambos tipos de alteraciones. El rendimiento en las tareas neuropsicológicas y el perfil psicopatológico de los participantes resultaron independientes.

En otro estudio (Nixon, Hallford, \& Tivis, 1996) se comparó la ejecución en tareas neuropsicológicas de un grupo de participantes con adicción al alcohol, un grupo de sujetos con esquizofrenia, y un grupo de participantes con es- 
quizofrenia y dependencia al alcohol. Aunque la hipótesis inicial del estudio apuntaba hacia la presencia de mayores déficits neuropsicológicos en el grupo dual, no hubo diferencias entre este grupo y el grupo de sujetos con esquizofrenia sin historia de dependencia de alcohol. No se encontraron efectos aditivos de la dependencia al alcohol sobre el rendimiento neuropsicológico de sujetos con esquizofrenia.

La investigación sobre la relación entre las alteraciones psicopatológicas y neuropsicológicas en la adicción a la cocaína podría contribuir al avance en el conocimiento de los mecanismos implicados en el inicio y en la evolución de esta adicción, y facilitar un mayor ajuste en la temporalización y en la selección de los procedimientos terapéuticos más adecuados en el tratamiento de esta adicción (Verdejo-García, López-Torrecillas, Gimenez, \& Pérez-García, 2004).

El objetivo del presente estudio consistió en examinar el estado psicopatológico de sujetos adictos a la cocaína al inicio de un programa de tratamiento y valorar en qué medida la presencia de estas alteraciones psicopatológicas se relacionaba con alteraciones neuropsicológicas. El estudio de esta relación se plantea en nuestro trabajo como una pregunta empírica, y no como una hipótesis, debido al limitado número de estudios previos entorno a la relación entre psicopatología y funcionamiento ejecutivo y a la naturaleza de los resultados encontrados.

\section{Método}

\section{Participantes}

En el estudio participaron 50 sujetos con adicción a la cocaína que habían solicitado voluntariamente asistencia terapéutica en el programa ambulatorio específico para la adicción a la cocaína de Proyecto Hombre de Asturias.

Los criterios de inclusión de los participantes fueron ser mayor de 20 años; cumplir los criterios de dependencia de la cocaína exclusiva o politoxicomanía con adicción principal a la cocaína según el DSM-IV-TR (American Psychiatric Association, 2000); no presentar trastornos psicopatológicos graves (demencia o psicosis); y presentar un período de abstinencia de cocaína mínima de una semana y máxima de un mes en el momento de la evaluación, con el fin de evitar la posible influencia de síntomas de deprivación o intoxicación.

En la Tabla 1 se presentan las principales características sociodemográficas y la historia y el patrón de consumo de drogas de la muestra.

Se trata en su mayoría de hombres entorno a los 30 años de edad, con estudios básicos, solteros (70\%), sin problemas médicos crónicos que interfieran con su vida (68\%), que nunca han estado en prisión ( $82 \%$ ) y que en el momento de inicio de tratamiento se encontraban activos $(56 \%)$ o inacti$\operatorname{vos}(42 \%)$ laboralmente.
Tabla 1. Características sociodemográficas y de consumo de la muestra ( $N$ $=50)$

\begin{tabular}{ll}
\hline Variables & Puntuaciones \\
\hline Sexo (\% hombres) & 94 \\
Edad, años $^{\mathrm{a}}$ & $29.8 \pm 5.7$ \\
Educación, años de escolarización $^{\mathrm{a}}$ & $9.61 \pm 2.6$ \\
Alguna vez en prisión (\%) & 18 \\
Activos laboralmente (\%) & 56 \\
Problemas médicos crónicos (\%) & 32 \\
Años de consumo regular de cocaína ${ }^{\mathrm{a}}$ & $7.52 \pm 5.41$ \\
Vía de administración nasal (\%) & 92 \\
Vía de administración fumada (\%) & 8 \\
Alcohol & 93.9 \\
Cannabis & 61.2 \\
MASTa & $6.14 \pm 4.7$ \\
Toma de disulfiram (\%) & 38 \\
\hline a $M \pm D T$ b Tres o más veces por semana, binges, o consumo irregular pro- \\
blemático que interfiere en el funcionamiento diario
\end{tabular}

\section{Instrumentos}

Todos los sujetos fueron evaluados mediante la aplicación de los criterios diagnósticos de dependencia a la cocaína del DSM-IV-TR (American Psychiatric Association, 2000); el instrumento European Addiction Severity Index (EuropASI; Kokkevi \& Hartgers, 1995) que proporciona información sobre seis áreas independientes: situación médica, situación laboral, consumo de alcohol/otras drogas, problemas legales, relaciones familiares/sociales, y estado psiquiátrico y en el que se utilizó la denominada puntuación compuesta (composite score) del estado psiquiátrico como variable psicopatológica; el Michigan Alcoholism Screening Test (MAST; Selzer, 1971) para la detección de abuso de alcohol y los problemas relacionados; el cuestionario Beck Depression Inventory (BDI; Beck, Ward, Mendelson, Mock, \& Erbaugh, 1961) para evaluar un amplio espectro de síntomas depresivos, en el que se han utilizado los puntos de corte usualmente aceptados (Beck, Steer, \& Garbin, 1988): no síntomas de depresión de 0 a 9, sintomatología leve de 10 a 18, sintomatología moderada de 19-29 y sintomatología grave más de 30 puntos; y el cuestionario autoaplicado Symptom Checklist 90 Revised (SCL-90-R; Derogatis, Lipman, \& Covi, 1973) que explora un amplio rango de problemas psicológicos y síntomas psicopatológicos y dispone de nueve dimensiones sintomáticas primarias de salud mental. Las nueve dimensiones son: somatizaciones (SOM), obsesiones y compulsiones (OBS), sensibilidad interpersonal (INT), depresión (DEP), ansiedad (ANS), hostilidad (HOS), ansiedad fóbica (FOB), ideación paranoide (PAR) y psicoticismo (PSI). Las puntuaciones directas convertidas en puntuaciones centiles iguales o superiores a 90 en cada una de las escalas, indican riesgo psicopatológico (Derogatis, 2002).

El protocolo de evaluación neuropsicológica se compuso de varias tareas, seleccionadas de distintos test, y sensibles a la detección de alteraciones en diferentes componentes del funcionamiento ejecutivo, incluyendo: atención, flexibilidad mental/memoria de trabajo, inhibición de respuestas automatizadas y fluidez (procesos de iniciación de respuestas). Se 
aplicó el Test de Palabras y Colores de Stroop (STROOP; Golden, 1994) que consta de tres partes y evalúa la capacidad de un sujeto para inhibir una respuesta automática, la habilidad de atención dividida y la resistencia a la interferencia; un Test de Programas Alternantes (paradigma go/no go) con un formato creado experimentalmente para medir control inhibitorio e interferencia atencional; el Test del Trazado o Trail Making Test de la Batería Neuropsicológica de Halstead-Reitan (TMT; Reitan \& Wolfson, 1985) que consta de dos partes y evalúa atención visual sostenida, atención alternante, flexibilidad mental, rastreo visual, velocidad de procesamiento de la información y habilidad grafomotora; y por último, el subtest de Evocación Categorial del Programa Integrado de Exploración Neuropsicológica "Test Barcelona" (PIEN; Peña-Casanova, 1991), prueba de fluidez verbal que consta de dos tareas y requiere de un adecuado funcionamiento de la memoria de trabajo y de las funciones ejecutivas, en especial de la iniciación de respuestas y de las estrategias de búsqueda.

Todos los instrumentos de evaluación utilizados en este estudio han mostrado buenas propiedades psicométricas en términos de fiabilidad y validez en múltiples estudios. En concreto, el EuropASI es uno de los instrumentos más utilizados en la investigación sobre drogodependencias y su versión española ha mostrado un buen rendimiento psicométrico (Díaz Mesa et al., 2010). Además, el uso de puntuaciones compuestas en lugar de la escala de gravedad del evaluador disminuye el sesgo subjetivo que se deriva de puntuaciones no estandarizadas (Sánchez-Hervás, Secades-Villa, Santonja Gómez, Zacarés Romaguera, \& García-Rodríguez, 2009). Tanto el MAST como el BDI son instrumentos diagnósticos frecuentemente utilizados en investigación que vienen avalados por sus buenas propiedades psicométricas tanto en población general como clínica (Beck, et al., 1988; Shields, Howell, Potter, \& Weiss, 2007). Por su parte, el SCL-90-R es uno de los instrumentos de evaluación de sintomatología psicopatológica más utilizados en estudios con adictos a sustancias mostrando excelentes índices de fiabilidad y validez en su versión española (Derogatis, 2002).

Las tareas utilizadas para la valoración neuropsicológica también son instrumentos de evaluación clínica frecuentemente utilizados en investigación. El test Stroop cuenta con baremos propios en nuestro país (Golden, 1994), al igual que el subtest de Evocación Categorial (Benito-Cuadrado, Esteba-Castillo, Bohm, Cejudo-Bolivar, \& Pena-Casanova, 2002) y el Trail Making Test sobre el que comienzan a aparecer datos normativos con muestras españolas (Perianez et al., 2007) que complementan los baremos de población americana (Tombaugh, 2004). Todas estas pruebas han mostrado buenos indicadores de fiabilidad test-retest, sensibilidad y especificidad (Peña-Casanova, Gramunt Fombuena, \& Gich Fullá, 2006).

Además, se utilizó el test de detección de cocaína en la orina de Perfelena para descartar consumos de cocaína y corroborar la abstinencia. Los niveles de corte que detectan estos test son los recomendados por el Substance Abuse and
Mental Health Services Administration (SAMHSA) del National Institute of Drug Abuse (NIDA) en Estados Unidos.

\section{Procedimiento}

Los participantes fueron seleccionados aleatoriamente del total de demandantes de tratamiento en el programa ambulatorio específico para la adicción a la cocaína de Proyecto Hombre de Asturias. Los sujetos fueron evaluados individualmente por personal cualificado durante dos sesiones en el marco de la evaluación pretratamiento. En la primera sesión, con una duración aproximada de 2 horas, los participantes fueron informados acerca del protocolo de la investigación, se evaluó el cumplimiento de los criterios diagnósticos DSM-IV-TR para la dependencia a la cocaína (American Psychiatric Association, 2000) y se aplicaron los instrumentos de evaluación EuropASI, MAST, BDI y SCL-90-R. Todos los participantes seleccionados dieron su consentimiento informado para participar en el estudio. El protocolo de evaluación neuropsicológico fue aplicado durante una segunda y única sesión de evaluación de una hora de duración en la que el orden de administración de las pruebas fue: Stroop, Test de Programas Alternantes, TMT y subtest de Evocación Categorial "Test Barcelona".

La evaluación de los pacientes tuvo lugar una vez transcurrido un período de abstinencia mínimo de 1 semana y máximo de 1 mes, con el objetivo de controlar la interferencia de posibles estados de intoxicación aguda o de sintomatología asociada a la abstinencia, así como para reducir la variabilidad en el tiempo de abstinencia de los participantes en el momento de aplicación de las pruebas.

A pesar de que la APA recomienda llevar a cabo la evaluación transcurridas 4 semanas de abstinencia (American Psychiatric Association, 2000), en la misma línea que estudios previos (Kokkevi, Stefanis, Anastasopoulou, \& Kostogianni, 1998), resulta de interés realizar una evaluación inicial temprana para conocer qué características y dificultades sufren los participantes al inicio de los programas de tratamiento para manejarlos de forma eficaz (Satel, Kosten, Schuckit, \& Fischman, 1993). Estudios previos utilizan criterios temporales de abstinencia de menor a mayor duración para explorar la evolución de las alteraciones neuropsicológicas a lo largo de períodos más largos de abstinencia (Bell, Foxe, Nierenberg, Hoptman, \& Garavan, 2011; García Fernández, et al., 2010; Schrimsher \& Parker, 2008).

\section{Análisis de datos}

Se realizaron análisis descriptivos y de frecuencias para describir las características sociodemográficas de la muestra y para analizar el patrón de consumo y el perfil psicopatológico de la muestra de adictos a la cocaína.

Para analizar la relación entre las variables psicopatológicas y las variables neuropsicológicas de la muestra se llevaron a cabo correlaciones: coeficiente de correlación de Pearson para variables cuantitativas con distribución normal y coeficiente de correlación de Spearman para las variables 
que no se distribuían normalmente. Finalmente, se aplicó la prueba $t$ de Student para comparar el rendimiento neuropsicológico de la muestra en función del riesgo psicopatológico. Todos los datos fueron analizados utilizando el paquete estadístico SPSS15.

\section{Resultados}

\section{Comorbilidad psicopatológica}

En la Tabla 2 se presentan las puntuaciones directas medias de la muestra en cada una de las escalas del SCL-90-R y sus puntuaciones centiles equivalentes, extraídas de la muestra normativa de la población general. Las puntuaciones medias en cada una de las escalas son superiores a las puntuaciones correspondientes al percentil 80 de la población general. Estos resultados indican que el grupo de adictos a la cocaína muestran una sintomatología psicopatológica más elevada que el $80 \%$ de la muestra general en todas las dimensiones sintomáticas evaluadas.

Tabla 2. Puntuaciones de la muestra clínica en el SCL-90-R.

\begin{tabular}{lcc}
\hline Escalas sintomáticas SCL-90-R & $\begin{array}{c}\text { Puntuaciones } \\
\text { directas }^{\mathrm{a}}\end{array}$ & $\begin{array}{c}\text { Puntuaciones } \\
\text { centiles }\end{array}$ \\
\hline Somatizaciones (SOM) & $0.68 \pm 0.79$ & 85 \\
Obsesiones-compulsiones (OBS) & $1.28 \pm 0.92$ & 90 \\
Sensibilidad interpersonal (INT) & $0.81 \pm 0.74$ & 80 \\
Depresión (DEP) & $1.07 \pm 0.92$ & 85 \\
Ansiedad (ANS) & $0.79 \pm 0.78$ & 85 \\
Hostilidad (HOS) & $0.87 \pm 0.93$ & 80 \\
Ansiedad fóbica (FOB) & $0.45 \pm 0.68$ & 80 \\
Ideación paranoide (PAR) & $0.96 \pm 0.89$ & 85 \\
Psicoticismo (PSI) & $0.58 \pm 0.71$ & 85 \\
\hline
\end{tabular}

a $M \pm D T$

En la Tabla 3 se presenta el porcentaje de sujetos en riesgo psicopatológico en cada una de las escalas del SCL90-R, las puntuaciones medias en el BDI y el nivel de gravedad de la sintomatología depresiva, y el composite score psiquiá- trico. Al inicio de tratamiento, el 26\% o más de los participantes puntuaban por encima del punto de corte de riesgo psicopatológico establecido (percentil 90) en cada una de las escalas del SCL-90-R y más de la mitad (54\%) superaba el punto de corte en la escala de obsesiones y compulsiones. Además, el $54 \%$ de los adictos a la cocaína presentaba síntomas depresivos en el momento de la evaluación. La mayoría síntomas depresivos leves (34.7\%) o moderados $(30.6 \%)$.

Tabla 3. Resultados en el SCL-90-R, BDI y área psiquiátrica del EuropASI. Variables Puntuación

Escalas sintomáticas SCL-90-R ${ }^{a}$

Somatizaciones (SOM) 26

Obsesiones y compulsiones (OBS) $\quad 54$

Sensibilidad interpersonal (INT) 32

Depresión (DEP) $\quad 40$

Ansiedad (ANS) 34

Hostilidad (HOS) 28

Ansiedad fóbica (FOB) 30

Ideación paranoide (PAR) $\quad 38$

Psicoticismo (PSI) 32

Gravedad BDI $(\%)$

$\leq 9$ No depresión $\quad 18.4$

10-18 Depresión leve $\quad 34.7$

19-29 Depresión moderada $\quad 30.6$

$\geq 30$ Depresión grave $\quad 16.3$

$\mathrm{BDI}^{\mathrm{b}}$

$18.3 \pm 10$

Composite score psiquiátrico $(\mathrm{CSP})^{\mathrm{b}}$

$0.28 \pm 0.27$

a $\%$ de sujetos con puntuaciones por encima del punto de corte de riesgo psicopatológico (Pctl. $\geq 90)$ b $M \pm D T$

\section{Relación entre perfil psicopatológico y neuropsi- cológico}

En la Tabla 4 se muestra la relación entre variables de índole psicopatológica (puntuaciones en el BDI, composite score del estado psiquiátrico del EuropASI y SCL-90-R), y la ejecución en las distintas pruebas de la batería neuropsicológica.

Tabla 4. Correlación bivariada entre variables psicopatológicas y neuropsicológicas.

\begin{tabular}{|c|c|c|c|c|c|c|c|c|c|c|c|}
\hline & \multirow[t]{2}{*}{ BDI } & \multirow[t]{2}{*}{ CS PSIQ. } & \multicolumn{6}{|c|}{ SCL-90-R } & \multirow[b]{2}{*}{ FOB } & \multirow[b]{2}{*}{ PAR } & \multirow[b]{2}{*}{ PSI } \\
\hline & & & SOM & OBS & INT & DEP & ANS & $\mathrm{HOS}$ & & & \\
\hline \multicolumn{12}{|l|}{ Test de Stroop } \\
\hline Palabras & .270 & .029 & .155 & .059 & -.091 & .112 & .175 & -.098 & -.138 & -.091 & -.121 \\
\hline Colores & .125 & -.047 & .078 & .002 & -.115 & .059 & .080 & -.033 & -.154 & .037 & -.024 \\
\hline Palabras y Colores & -.079 & -.036 & .086 & .051 & -.139 & .086 & -.041 & -.041 & -.024 & -.096 & .131 \\
\hline Índice interferencia & -.259 & -.033 & -.118 & .030 & -.061 & .021 & -.168 & -.094 & .000 & -.098 & .075 \\
\hline \multicolumn{12}{|c|}{ Test de Programas Alternantes } \\
\hline Errores & .015 & -.040 & .007 & -.029 & .090 & .026 & .062 & -.058 & .136 & -.032 & .004 \\
\hline Autocorrecciones & .078 & -.013 & -.129 & .098 & .213 & -.050 & .042 & .079 & -.057 & .214 & .155 \\
\hline \multicolumn{12}{|l|}{ Test del Trazado } \\
\hline Parte A.- Tiempo & -.045 & .023 & .153 & .259 & .007 & .271 & .220 & .198 & $.344^{*}$ & .079 & .267 \\
\hline Errores & -.013 & .002 & .100 & .087 & -.072 & .078 & .023 & -.084 & .081 & -.043 & .063 \\
\hline Parte B.- Tiempo & -.063 & .064 & .133 & .248 & .133 & $.299 *$ & .108 & .151 & .213 & .066 & .176 \\
\hline Errores & -.149 & -.199 & -.022 & -.061 & .057 & .033 & .010 & -.063 & .052 & .053 & .119 \\
\hline \multicolumn{12}{|c|}{ Pruebas de Fluidez Verbal } \\
\hline Animales & .042 & -.213 & .172 & .148 & -.051 & .086 & .074 & .133 & -.023 & .199 & .204 \\
\hline Palabras P & .136 & -.024 & -.054 & .110 & -.128 & .162 & .022 & -.081 & -.157 & -.071 & -.022 \\
\hline
\end{tabular}


Se encontró una relación estadísticamente significativa entre las escala depresión (DEP) del SCL-90-R y el tiempo empleado en la parte B del Test del Trazado $(\phi<.05)$ y entre la escala ansiedad fóbica (FOB) del SCL-90-R y el tiempo empleado en la parte A del Test del Trazado $(p<.05)$. El grado de asociación es medio y con sentido positivo. Es decir, un mayor grado de depresión o ansiedad fóbica se relaciona con el aumento del tiempo necesario para realizar las tareas del Test del Trazado. En el resto de las variables psicopatológicas y neuropsicológicas evaluadas no se encontraron relaciones estadísticamente significativas.

En la Tabla 5 se muestra la relación entre las puntuaciones medias obtenidas en la batería neuropsicológica en función del punto de corte de riesgo psicopatológico del SCL90-R. Se comparó la ejecución en cada una de las tareas neu- ropsicológicas del grupo de sujetos en riesgo psicopatológico frente al grupo de participantes que se situaban por debajo de la puntuación de riesgo en cada una de las escalas sintomáticas. Los resultados mostraron diferencias estadísticamente significativas $(p<.05)$ en la ejecución de la parte A del Test del Trazado en relación a la escala depresión (DEP), y en el número de autocorrecciones en el Test de Programas Alternantes en relación a la escala hostilidad (HOS). El rendimiento en la parte A del Test del Trazado es mejor en el grupo de sujetos que se encuentra en riesgo psicopatológico en la escala de depresión, y la puntuación en el Test de Programas Alternantes (número de autocorrecciones) es mejor en el grupo de sujetos que no se encuentran en riesgo psicopatológico en la escala hostilidad.

Tabla 5. Diferencias en la ejecución neuropsicológica en función del riesgo psicopatológico en las escalas del SCL-90-R.

\begin{tabular}{|c|c|c|c|c|c|c|c|c|c|c|c|c|c|c|c|c|c|c|}
\hline & \multicolumn{2}{|c|}{ SOM } & \multicolumn{2}{|c|}{ OBS } & \multicolumn{2}{|c|}{ INT } & \multicolumn{2}{|c|}{ DEP } & \multicolumn{2}{|c|}{ ANS } & \multicolumn{2}{|c|}{ PSI } & \multicolumn{2}{|c|}{ HOS } & \multicolumn{2}{|c|}{ FOB } & \multicolumn{2}{|c|}{ PAR } \\
\hline & GRP & GRNP & GNP & GNRP & GRP & GNRP & GRP & GNRP & GRP & GNRP & GRP & GNRP & GRP & GNRP & GRP & GRNP & GRP & GNRP \\
\hline \multicolumn{19}{|l|}{ Test de Stroop ${ }^{a}$} \\
\hline Palabras & $\begin{array}{c}103.80 \\
(16.00)\end{array}$ & $\begin{array}{l}112.00 \\
(16.05)\end{array}$ & $\begin{array}{l}109.17 \\
(14.43)\end{array}$ & $\begin{array}{l}103.22 \\
(18.99)\end{array}$ & $\begin{array}{l}108.85 \\
(13.88)\end{array}$ & $\begin{array}{r}99.81 \\
(21.84)\end{array}$ & $\begin{array}{l}104.23 \\
(15.80)\end{array}$ & $\begin{array}{l}108.55 \\
(19.11)\end{array}$ & $\begin{array}{l}106.09 \\
(16.63)\end{array}$ & $\begin{array}{l}105.70 \\
(18.62)\end{array}$ & $\begin{array}{l}106.02 \\
(15.71)\end{array}$ & $\begin{array}{l}105.78 \\
(21.06)\end{array}$ & $\begin{array}{l}104.54 \\
(15.83)\end{array}$ & $\begin{array}{l}109.26 \\
(20.09)\end{array}$ & $\begin{array}{l}107.35 \\
(14.30)\end{array}$ & $\begin{array}{l}103.68 \\
(21.22)\end{array}$ & $\begin{array}{l}106.02 \\
(16.16)\end{array}$ & $\begin{array}{l}105.81 \\
(19.64)\end{array}$ \\
\hline Colores & $\begin{array}{l}68.18 \\
(10.39)\end{array}$ & $\begin{array}{r}76.58 \\
(15.38)\end{array}$ & $\begin{array}{l}72.00 \\
(9.34)\end{array}$ & $\begin{array}{r}70.18 \\
(14.02)\end{array}$ & $\begin{array}{r}72.21 \\
(12.35)\end{array}$ & $\begin{array}{r}68.50 \\
(11.41)\end{array}$ & $\begin{array}{l}69.53 \\
(10.34)\end{array}$ & $\begin{array}{r}73.31 \\
(14.36)\end{array}$ & $\begin{array}{c}70.96 \\
(11.90)\end{array}$ & $\begin{array}{r}71.01 \\
(12.72)\end{array}$ & $\begin{array}{r}71.20 \\
(12.50)\end{array}$ & $\begin{array}{r}70.50 \\
(11.31)\end{array}$ & $\begin{array}{c}70.26 \\
(11.19)\end{array}$ & $\begin{array}{r}72.66 \\
(14.11)\end{array}$ & $\begin{array}{r}69.38 \\
(10.95)\end{array}$ & $\begin{array}{r}73.77 \\
(13.64)\end{array}$ & $\begin{array}{r}70.54 \\
(11.44)\end{array}$ & $\begin{array}{r}71.93 \\
(13.59)\end{array}$ \\
\hline Palabras y Colores & $\begin{array}{l}44.32 \\
(9.31)\end{array}$ & $\begin{array}{c}45.83 \\
(9.44)\end{array}$ & $\begin{array}{l}45.45 \\
(9.54)\end{array}$ & $\begin{array}{l}44.07 \\
(9.17)\end{array}$ & $\begin{array}{l}45.57 \\
(10.27)\end{array}$ & $\begin{array}{l}42.87 \\
(6.69)\end{array}$ & $\begin{array}{l}43.70 \\
(9.81)\end{array}$ & $\begin{array}{l}46.26 \\
(8.35)\end{array}$ & $\begin{array}{l}45.43 \\
(8.71)\end{array}$ & $\begin{array}{r}43.29 \\
(10.37)\end{array}$ & $\begin{array}{l}45.05 \\
(9.90)\end{array}$ & $\begin{array}{l}43.78 \\
(7.72)\end{array}$ & $\begin{array}{l}44.55 \\
(9.79)\end{array}$ & $\begin{array}{l}45.00 \\
(8.28)\end{array}$ & $\begin{array}{l}45.25 \\
(9.09)\end{array}$ & $\begin{array}{l}43.72 \\
(9.74)\end{array}$ & $\begin{array}{l}44.45 \\
(9.52)\end{array}$ & $\begin{array}{l}45.18 \\
(8.99)\end{array}$ \\
\hline Índice interferencia & $\begin{array}{l}3.06 \\
(8.22)\end{array}$ & $\begin{array}{l}0.70 \\
(6.60)\end{array}$ & $\begin{array}{r}2.36 \\
(9.56)\end{array}$ & $\begin{array}{r}2.58 \\
(6.32)\end{array}$ & $\begin{array}{l}2.48 \\
(8.90)\end{array}$ & $\begin{array}{r}2.47 \\
(5.32)\end{array}$ & $\begin{array}{l}2.28 \\
(9.11)\end{array}$ & $\begin{array}{r}2.79 \\
(5.54)\end{array}$ & $\begin{array}{c}3.31 \\
(7.58)\end{array}$ & $\begin{array}{r}0.92 \\
(8.35)\end{array}$ & $\begin{array}{l}2.84 \\
(8.54)\end{array}$ & $\begin{array}{r}1.59 \\
(6.00)\end{array}$ & $\begin{array}{l}2.90 \\
(8.82)\end{array}$ & $\begin{array}{r}1.54 \\
(5.16)\end{array}$ & $\begin{array}{r}3.31 \\
(8.41)\end{array}$ & $\begin{array}{r}1.05 \\
(6.78)\end{array}$ & $\begin{array}{r}2.44 \\
(8.52)\end{array}$ & $\begin{array}{r}2.56 \\
(6.52)\end{array}$ \\
\hline \multicolumn{3}{|c|}{ Test de Programas Alternantes a } & & & & & & & & & & & & & & & & \\
\hline Errores & $\begin{array}{c}0.35 \\
(0.94)\end{array}$ & $\begin{array}{r}0.15 \\
(0.37)\end{array}$ & $\begin{array}{r}0.21 \\
(0.42)\end{array}$ & $\begin{array}{r}0.37 \\
(1.07)\end{array}$ & $\begin{array}{l}0.35 \\
(0.98)\end{array}$ & $\begin{array}{r}0.18 \\
(0.40)\end{array}$ & $\begin{array}{r}0.26 \\
(0.78)\end{array}$ & $\begin{array}{r}0.35 \\
(0.93)\end{array}$ & $\begin{array}{c}0.12 \\
(0.33)\end{array}$ & $\begin{array}{r}0.64 \\
(1.32)\end{array}$ & $\begin{array}{c}0.33 \\
(0.95)\end{array}$ & $\begin{array}{r}0.21 \\
(0.42)\end{array}$ & $\begin{array}{l}0.34 \\
(0.96)\end{array}$ & $\begin{array}{l}0.20 \\
(0.41)\end{array}$ & $\begin{array}{l}0.19 \\
(0.40)\end{array}$ & $\begin{array}{r}0.47 \\
(1.26)\end{array}$ & $\begin{array}{c}0.29 \\
(0.75)\end{array}$ & $\begin{array}{r}0.31 \\
(1.01)\end{array}$ \\
\hline Autocorrecciones & $\begin{array}{l}1.59 \\
(1.44)\end{array}$ & $\begin{array}{l}1.46 \\
(0.96) \\
\end{array}$ & $\begin{array}{l}1.39 \\
(1.33)\end{array}$ & $\begin{array}{r}1.70 \\
(1.32)\end{array}$ & $\begin{array}{l}1.47 \\
(1.37)\end{array}$ & $\begin{array}{r}1.75 \\
(1.23)\end{array}$ & $\begin{array}{l}1.66 \\
(1.56)\end{array}$ & $\begin{array}{r}1.40 \\
(0.88)\end{array}$ & $\begin{array}{l}1.60 \\
(1.49)\end{array}$ & $\begin{array}{l}1.47 \\
(0.94)\end{array}$ & $\begin{array}{c}1.61 \\
(1.41)\end{array}$ & $\begin{array}{r}1.42 \\
(1.08)\end{array}$ & $\begin{array}{l}1.77^{*} \\
(1.45)\end{array}$ & $\begin{array}{l}1.06 * \\
(0.79)\end{array}$ & $\begin{array}{l}1.45 \\
(1.33)\end{array}$ & $\begin{array}{r}1.73 \\
(1.32)\end{array}$ & $\begin{array}{l}1.52 \\
(1.46)\end{array}$ & $\begin{array}{r}1.62 \\
(1.02)\end{array}$ \\
\hline \multicolumn{19}{|l|}{ Test del Trazado a } \\
\hline $\begin{array}{l}\text { Parte A } \\
\text { Tiempo }\end{array}$ & $\begin{array}{l}27.27 \\
(9.64)\end{array}$ & $\begin{array}{l}28.53 \\
(9.58)\end{array}$ & $\begin{array}{c}25.21 \\
(7.76)\end{array}$ & $\begin{array}{r}29.62 \\
(10.55)\end{array}$ & $\begin{array}{l}27.50 \\
(9.77)\end{array}$ & $\begin{array}{l}27.81 \\
(9.36)\end{array}$ & $\begin{array}{l}25.26^{*} \\
(8.19)\end{array}$ & $\begin{array}{c}31.10^{*} \\
(10.53)\end{array}$ & $\begin{array}{c}26.06 \\
(9.28)\end{array}$ & $\begin{array}{l}30.58 \\
(9.61)\end{array}$ & $\begin{array}{c}26.91 \\
(9.44)\end{array}$ & $\begin{array}{l}29.35 \\
(9.92)\end{array}$ & $\begin{array}{c}26.42 \\
(8.15)\end{array}$ & $\begin{array}{r}30.33 \\
(12.08)\end{array}$ & $\begin{array}{l}27.90 \\
(10.57)\end{array}$ & $\begin{array}{c}27.10 \\
(7.83)\end{array}$ & $\begin{array}{c}26.64 \\
(9.59)\end{array}$ & $\begin{array}{l}29.62 \\
(9.41)\end{array}$ \\
\hline Errores & $\begin{array}{l}0.16 \\
(0.44)\end{array}$ & $\begin{array}{r}0.15 \\
(0.37)\end{array}$ & $\begin{array}{r}0.08 \\
(0.28)\end{array}$ & $\begin{array}{r}0.22 \\
(0.50)\end{array}$ & $\begin{array}{r}0.14 \\
(0.35)\end{array}$ & $\begin{array}{c}0.18 \\
(0.54)\end{array}$ & $\begin{array}{r}0.10 \\
(0.30)\end{array}$ & $\begin{array}{r}0.25 \\
(0.55)\end{array}$ & $\begin{array}{r}0.15 \\
(0.36)\end{array}$ & $\begin{array}{c}0.17 \\
(0.52)\end{array}$ & $\begin{array}{r}0.19 \\
(0.46)\end{array}$ & $\begin{array}{r}0.07 \\
(0.26)\end{array}$ & $\begin{array}{l}0.05 \\
(0.23)\end{array}$ & $\begin{array}{c}0.40 \\
(0.63)\end{array}$ & $\begin{array}{r}0.19 \\
(0.47)\end{array}$ & $\begin{array}{r}0.10 \\
(0.31)\end{array}$ & $\begin{array}{c}0.11 \\
(0.32)\end{array}$ & $\begin{array}{r}0.25 \\
(0.57)\end{array}$ \\
\hline $\begin{array}{l}\text { Parte B } \\
\text { Tiempo }\end{array}$ & $\begin{array}{r}65.32 \\
(25.14)\end{array}$ & $\begin{array}{c}69.53 \\
(35.06)\end{array}$ & $\begin{array}{c}60.78 \\
(19.94)\end{array}$ & $\begin{array}{r}71.22 \\
(32.56)\end{array}$ & $\begin{array}{l}64.41 \\
(24.76)\end{array}$ & $\begin{array}{r}70.68 \\
(33.67)\end{array}$ & $\begin{array}{c}62.23 \\
(21.07)\end{array}$ & $\begin{array}{r}72.70 \\
(35.16)\end{array}$ & $\begin{array}{c}66.24 \\
(25.47)\end{array}$ & $\begin{array}{r}66.76 \\
(32.51)\end{array}$ & $\begin{array}{r}63.50 \\
(24.42)\end{array}$ & $\begin{array}{r}73.92 \\
(34.76)\end{array}$ & $\begin{array}{l}64.11 \\
(23.97)\end{array}$ & $\begin{array}{r}71.80 \\
(35.37)\end{array}$ & $\begin{array}{r}68.45 \\
(31.45)\end{array}$ & $\begin{array}{r}63.10 \\
(20.63)\end{array}$ & $\begin{array}{c}62.32 \\
(22.56)\end{array}$ & $\begin{array}{r}75.12 \\
(35.68)\end{array}$ \\
\hline Errores & $\begin{array}{c}0.35 \\
(0.71) \\
\end{array}$ & $\begin{array}{r}0.30 \\
(0.48) \\
\end{array}$ & $\begin{array}{c}0.34 \\
(0.77)\end{array}$ & $\begin{array}{r}0.33 \\
(0.55)\end{array}$ & $\begin{array}{c}0.35 \\
(0.69)\end{array}$ & $\begin{array}{c}0.31 \\
(0.60)\end{array}$ & $\begin{array}{r}0.30 \\
(0.70)\end{array}$ & $\begin{array}{r}0.40 \\
(0.35)\end{array}$ & $\begin{array}{c}0.39 \\
(0.74)\end{array}$ & $\begin{array}{r}0.23 \\
(0.43)\end{array}$ & $\begin{array}{l}0.36 \\
(0.68)\end{array}$ & $\begin{array}{c}0.28 \\
(0.61)\end{array}$ & $\begin{array}{r}0.34 \\
(0.75)\end{array}$ & $\begin{array}{c}0.33 \\
(0.48)\end{array}$ & $\begin{array}{c}0.32 \\
(0.70)\end{array}$ & $\begin{array}{r}0.36 \\
(0.59)\end{array}$ & $\begin{array}{r}0.32 \\
(0.68)\end{array}$ & $\begin{array}{r}0.37 \\
(0.61)\end{array}$ \\
\hline $\begin{array}{l}\text { Fluidez Verbal a } \\
\text { Animales }\end{array}$ & $\begin{array}{l}23.67 \\
(5.20)\end{array}$ & $\begin{array}{l}25.92 \\
(5.93)\end{array}$ & $\begin{array}{l}23.17 \\
(4.88)\end{array}$ & $\begin{array}{l}25.18 \\
(5.78)\end{array}$ & $\begin{array}{l}24.08 \\
(5.56)\end{array}$ & $\begin{array}{l}24.62 \\
(5.30)\end{array}$ & $\begin{array}{l}23.96 \\
(4.89)\end{array}$ & $\begin{array}{l}24.70 \\
(6.25)\end{array}$ & $\begin{array}{c}24.18 \\
(5.20)\end{array}$ & $\begin{array}{l}24.41 \\
(6.01)\end{array}$ & $\begin{array}{l}24.05 \\
(5.68)\end{array}$ & $\begin{array}{l}24.78 \\
(4.87)\end{array}$ & $\begin{array}{c}24.77 \\
(5.50)\end{array}$ & $\begin{array}{l}23.06 \\
(5.22)\end{array}$ & $\begin{array}{l}23.38 \\
(5.32)\end{array}$ & $\begin{array}{l}25.68 \\
(5.43)\end{array}$ & $\begin{array}{l}23.76 \\
(5.52)\end{array}$ & $\begin{array}{l}25.31 \\
(5.23)\end{array}$ \\
\hline Palabras $\mathbf{P}$ & $\begin{array}{c}33.10 \\
(9.18)\end{array}$ & $\begin{array}{r}35.00 \\
(12.52)\end{array}$ & $\begin{array}{l}32.65 \\
(8.23)\end{array}$ & $\begin{array}{r}34.40 \\
(11.46)\end{array}$ & $\begin{array}{c}35.35 \\
(11.08)\end{array}$ & $\begin{array}{l}29.87 \\
(6.15)\end{array}$ & $\begin{array}{l}32.10 \\
(7.83)\end{array}$ & $\begin{array}{r}35.85 \\
(12.57)\end{array}$ & $\begin{array}{l}33.87 \\
(9.49)\end{array}$ & $\begin{array}{r}33.05 \\
(11.33)\end{array}$ & $\begin{array}{c}34.41 \\
(11.01)\end{array}$ & $\begin{array}{r}31.50 \\
(6.92)\end{array}$ & $\begin{array}{c}34.28 \\
(10.73)\end{array}$ & $\begin{array}{c}32.00 \\
(8.35)\end{array}$ & $\begin{array}{c}33.48 \\
(9.08)\end{array}$ & $\begin{array}{l}33.78 \\
(11.72)\end{array}$ & $\begin{array}{l}32.41 \\
(10.11)\end{array}$ & $\begin{array}{l}36.12 \\
(9.75)\end{array}$ \\
\hline
\end{tabular}

\section{Discusión y conclusiones}

El objetivo de este estudio fue analizar la relación entre el estado psicopatológico y el perfil neuropsicológico de pacientes adictos a la cocaína al inicio de un programa de tratamiento y valorar en qué medida la mayor gravedad psicopatológica se relacionaba con una mayor presencia de déficit neuropsicológicos.

En un primer momento se realizó un acercamiento descriptivo a la caracterización psicopatológica de la muestra. La mayoría de los pacientes presentaban sintomatología depresiva leve y/o moderada y otras alteraciones psicopatoló- gicas. La presencia de alteraciones en el estado de ánimo se ha relacionado frecuentemente con la adicción a la cocaína y esta asociación se ha explicado frecuentemente en términos de semejanza entre síntomas depresivos y síntomas propios de la abstinencia a la cocaína, como consecuencia de las dificultades familiares, laborales y sociales generadas por la propia adicción, o como factor predisponente previo al trastorno adictivo (Brown et al., 1998; Herrero, Domingo-Salvany, Brugal, \& Torrens, 2011; López \& Becoña, 2007).

Parece conveniente evaluar el estado de ánimo durante la evaluación inicial y monitorizar los síntomas depresivos frecuentemente a lo largo del tratamiento con el fin de detectar los casos que requieran intervenciones complementarias. En 
este sentido, contamos con intervenciones psicológicas con evidencia contrastada que incluyen en su programa de tratamiento un protocolo de depresión en el que resulta necesario monitorizar el estado de ánimo desde el inicio del tratamiento para detectar aquellos casos en los que la sintomatología depresiva no evoluciona favorablemente tras las primeras semanas de abstinencia (Budney \& Higgins, 1998). No obstante, sería recomendable confirmar la estabilidad de estas puntuaciones más allá de las primeras semanas de abstinencia con el fin de poder diferenciar entre comorbilidad psicopatológica y los efectos directos del síndrome de abstinencia. Se requieren más investigaciones que analicen la repercusión de la sintomatología depresiva y psicopatológica sobre los resultados de tratamiento, y que exploren la evolución de esta sintomatología para determinar en qué casos desaparece o se mantiene a lo largo del tratamiento y qué variables median su evolución (Herrero, et al., 2011).

Además de la sintomatología depresiva, un elevado porcentaje de la muestra presenta también otras alteraciones psicopatológicas asociadas. La puntuación media de los participantes en todas las escalas del SCL-90-R fue superior a la media poblacional y el porcentaje de pacientes que superaron el punto de corte de riesgo psicopatológico fue igualmente muy elevado en todas las dimensiones sintomáticas. Además, en la dimensión de obsesiones y compulsiones (OBS) la puntuación centil de la muestra fue 90, lo que indica un posible riesgo para desarrollar trastornos de este espectro psicopatológico. Estos resultados son consistentes con los hallados en estudios previos (Fernández-Montalvo, Lorea, López-Goñi, \& Landa, 2008; Rosselli, et al., 2001; Sánchez Hervás, Tomas Gradoli, \& Morales Gallus, 2000), por lo que sería de interés continuar investigando el papel que juegan las alteraciones obsesivo-compulsivas en el inicio, mantenimiento y pronóstico de la adicción a la cocaína.

En este sentido, la adicción a drogas parece estar asociada con alteraciones en los sistemas responsables de la planificación, el control de la conducta y la regulación de las emociones, que facilitan la transición desde una fase inicial en la que las drogas se consumen por sus efectos reforzantes hacia una fase de dependencia en la que las conductas de consumo se transforman en rituales compulsivos que se mantienen a pesar de sus consecuencias negativas (Sociedad Española de Toxicomanías, 2011). Estudios acerca de otros problemas como la obesidad también apuntan hacia la implicación de estos mecanismos en la aparición de obsesiones y conductas compulsivas, en este caso relacionadas con la comida (Barry, Clarke, \& Petry, 2009).

Con respecto a la relación entre variables psicopatológicas y neuropsicológicas, los resultados mostraron que el tiempo empleado en el Test del Trazado en la parte A se relacionó con la escala de ansiedad fóbica (FOB) y la parte $\mathrm{B}$ con la escala depresión (DEP). Parece que la ansiedad se relaciona con la presencia de dificultades atencionales y de velocidad de procesamiento de información y los síntomas depresivos se relacionan con dificultades en la atención alternante y en la flexibilidad mental. Investigaciones previas han encontrado resultados en esta dirección, sin que se haya identificado una asociación robusta entre alteraciones psicopatológicas y rendimiento en tareas neuropsicológicas (Rosselli, et al., 2001). No se encontraron correlaciones significativas entre el resto de dimensiones psicopatológicas y los resultados de la batería neuropsicológica. No obstante, estos resultados han de tomarse con cautela ya que existe la posibilidad de que fueran requeridas medidas de la gravedad psicopatológica más específicas y sensibles.

Cuando se comparan las medias obtenidas por los pacientes en la batería neuropsicológica, en función de la presencia o ausencia de riesgo psicopatológico en las dimensiones del SCL-90-R, únicamente se encuentran diferencias estadísticamente significativas en el tiempo empleado en completar la tarea A del Test del trazado en la dimensión depresión (DEP) y en el número de autocorrecciones de la prueba de programas alternantes en la dimensión hostilidad (HOS). Los sujetos por debajo del riesgo psicopatológico en la dimensión hostilidad han obtenido mejores puntuaciones en la prueba de programas alternantes, pero los resultados en la tarea A del Test del Trazado en la dimensión depresión resultan inesperados, ya que los participantes con riesgo psicopatológico realizan mejor esta prueba. Se trata de una tarea sencilla perceptivo-motora y una menor dificultad en las tareas neuropsicológicas se ha relacionado en estudios previos con una mayor probabilidad de no encontrar diferencias o encontrar resultados dispares (Lorea, 2006).

Este estudio presenta algunas limitaciones que deben ser tenidas en cuenta. La exclusión de pacientes que presentaban trastornos psicopatológicos graves al inicio de tratamiento ha podido influir en los resultados obtenidos. Por este motivo, la generalización de estos resultados a la realidad clínica de adictos a la cocaína ha de realizarse con cautela puesto que podría infravalorarse la presencia e intensidad de las alteraciones neuropsicológicas y psicopatológicas asociadas a esta adicción.

El escaso tamaño de la muestra, la no consideración de algunas variables clínicas de interés (por ejemplo, variables de gravedad de la adicción tal como años de consumo, edad de inicio de consumo o el consumo de otras sustancias), la especificidad de los instrumentos utilizados para evaluar la psicopatología asociada con formato de autoinforme; y los problemas inherentes a la exploración de las funciones ejecutivas (García-Molina, Tirapu-Ustárroz, \& Roig-Rovira, 2007) son limitaciones que deberían intentar subsanarse en futuras investigaciones.

A pesar de estas limitaciones, los resultados de este estudio señalan que los adictos a la cocaína presentan alteraciones psicopatológicas por encima de la media poblacional al inicio de tratamiento, y que estas alteraciones no se asocian con un bajo rendimiento neuropsicológico general.

En la misma línea que otras investigaciones en las que se exploran las alteraciones neuropsicológicas ligadas a trastornos como los trastornos de la conducta alimentaria (Roberts, Tchanturia, Stahl, Southgate, \& Treasure, 2007), la esquizofrenia (Vázquez Valverde \& López Luengo, 2003) o la es- 
clerosis múltiple (Introzzi \& Ledesma, 2008), resultaría de interés explorar la evolución y la relación de las alteraciones neuropsicológicas y alteraciones psicopatológicas en la adicción a la cocaína a lo largo del tratamiento, y determinar qué variables pueden influir en la recuperación de este tipo de

\section{Referencias}

Aharonovich, E., Hasin, D. S., Brooks, A. C., Liu, X., Bisaga, A., \& Nunes, E. V. (2006). Cognitive deficits predict low treatment retention in cocaine dependent patients. Drug and Alcohol Dependence, 81(3), 313-322.

American Psychiatric Association. (2000). Diagnostic and statistical manual of mental disorders (4th ed. revised). Washington, DC: American Psychiatric Association.

Barry, D., Clarke, M., \& Petry, N. M. (2009). Obesity and its relationship to addictions: is overeating a form of addictive behavior? American Journal on Addictions, 18(6), 439-451.

Beck, A. T., Steer, R. A., \& Garbin, M. G. (1988). Psychometric properties of the Beck Depression Inventory. Twenty-five years of evaluation. Clinical Psychology Review, 8, 77-100.

Beck, A. T., Ward, C. H., Mendelson, M., Mock, J., \& Erbaugh, J. (1961). An inventory for measuring depression. Archives of General Psychiatry, 4, 561 571.

Bell, R. P., Foxe, J. J., Nierenberg, J., Hoptman, M. J., \& Garavan, H. (2011). Assessing white matter integrity as a function of abstinence duration in former cocaine-dependent individuals. Drug and Alcohol Dependence, 114(2-3), 159-168.

Benito-Cuadrado, M. M., Esteba-Castillo, S., Bohm, P., Cejudo-Bolivar, J., \& Pena-Casanova, J. (2002). Semantic verbal fluency of animals: a normative and predictive study in a Spanish population. Journal of Clinical and Experimental Neuropsychology, 24(8), 1117-1122.

Brown, R. A., Monti, P. M., Myers, M. G., Martin, R. A., Rivinus, T., Dubreuil, M. E., et al. (1998). Depression among cocaine abusers in treatment: relation to cocaine and alcohol use and treatment outcome. American Journal of Psychiatry, 155(2), 220-225.

Budney, A. J., \& Higgins, S. T. (1998). A Community Reinforcement Plus Vouchers Approach: Treating Cocaine Addiction. Rockville: National Institute on Drug Abuse.

Derogatis, L. R. (2002). SCL-90-R. Cuestionario de 90 sintomas. Madrid: TEA.

Derogatis, L. R., Lipman, R. S., \& Covi, L. (1973). SCL-90: an outpatient psychiatric rating scale--preliminary report. Psychopharmacology Bulletin, 9(1), 13-28.

Di Sclafani, V., Tolou-Shams, M., Price, L. J., \& Fein, G. (2002). Neuropsychological performance of individuals dependent on crackcocaine, or crack-cocaine and alcohol, at 6 weeks and 6 months of abstinence. Drug and Alcobol Dependence, 66(2), 161-171.

Díaz Mesa, E. M., García-Portilla, P., Saiz, P. A., Bobes Bascarán, T., Casares, M. J., Fonseca, E., et al. (2010). Psychometric performance of the 6th version of the Addiction Severity Index in Spanish (ASI-6). Psicothema, 22(3), 513-519.

Fernández-Montalvo, J., Lorea, I., López-Goñi, J. J., \& Landa, N. (2008). Comorbilidad psicopatológica en la adicción a la cocaína: resultados con el SCL-90-R. Psicología Conductual, 16(2), 273-286.

Fernández-Serrano, M. J., Pérez-García, M., Schmidt Río-Valle, J., \& Verdejo-García, A. (2010). Neuropsychological consequences of alcohol and drug abuse on different components of executive functions. Journal of Psychopharmacology, 24(9), 1317-1332.

Fernández-Serrano, M. J., Pérez-García, M., \& Verdejo-García, A. (2011). What are the specific vs. generalized effects of drugs of abuse on neuropsychological performance? Neuroscience and Biobehavioral Reviews, 35(3), 377-406.

García-Molina, A., Tirapu-Ustárroz, J., \& Roig-Rovira, T. (2007). Validez ecológica en la exploración de las funciones ejecutivas. Anales de Psicologia, 23(2), 289-299.

García Fernández, G., García Rodríguez, O., Secades Villa, R., Fernández Hermida, J. R., \& Sánchez Hervás, E. (2010). Evolución de las pacientes para desarrollar tratamientos cada vez más eficaces y ajustados a la realidad clínica.

Agradecimientos.- Agradecimientos a Proyecto Hombre Asturias. Este estudio forma parte de un proyecto de investigación financiado por la Delegación del Gobierno del Plan Nacional sobre Drogas (Ministerio de Sanidad y Consumo) con referencia MSC-06-01.

funciones ejecutivas de adictos a la cocaína tras un año de tratamiento. Revista Española de Drogodependencias, 35(2), 155-169.

Golden, C. J. (1994). STROOP. Manual del Test de Colores y Palabras. Madrid: TEA.

Herrero, M. J., Domingo-Salvany, A., Brugal, M. T., \& Torrens, M. (2011) Incidence of psychopathology in a cohort of young heroin and/or cocaine users. Journal of Substance Abuse Treatment, 41(1), 55-63.

Herrero, M. J., Domingo-Salvany, A., Torrens, M., \& Brugal, M. T. (2008). Psychiatric comorbidity in young cocaine users: induced versus independent disorders. Addiction, 103(2), 284-293.

Introzzi, I., \& Ledesma, R. D. (2008). Función ejecutiva y uso de estrategias semánticas en pacientes con esclerosis múltiple. Anales de Psicología, 24(2), 277-283.

Jovanovski, D., Erb, S., \& Zakzanis, K. K. (2005). Neurocognitive deficits in cocaine users: a quantitative review of the evidence. Journal of Clinical and Experimental Neuropsychology, 27(2), 189-204.

Kokkevi, A., \& Hartgers, C. (1995). European adaptation of a multidimensional assessment instrument for drug and alcohol dependence. European Addiction Research, 1, 208-210.

Kokkevi, A., Stefanis, N., Anastasopoulou, E., \& Kostogianni, C. (1998). Personality disorders in drug abusers: prevalence and their association with AXIS I disorders as predictors of treatment retention. Addictive Behaviors, 23(6), 841-853.

López, A., \& Becoña, E. (2007). Depression and cocaine dependence. Psychological Reports, 100(2), 520-524.

López Durán, A., \& Becoña Iglesias, E. (2006). Patterns and personality disorders in persons with cocaine dependence in treatment. Psicothema, 18(3), 578-583.

Lorea, I. (2006). Características clínicas, comorbilidad psicopatológica y rendimiento neuropsicológico en adictos a la cocaína en tratamiento ambulatorio: Universidad Pública de Navarra.

Lorea, I., Fernández-Montalvo, J., Tirapu-Ustarroz, J., Landa, N., \& LópezGoñi, J. J. (2010). Neuropsychological performance in cocaine addiction: a critical review. Revista de Neurología, 51(7), 412-426.

Nixon, S. J., Hallford, H. G., \& Tivis, R. D. (1996). Neurocognitive function in alcoholic, schizophrenic, and dually diagnosed patients. Psychiatry Research, 64(1), 35-45.

Pace-Schott, E. F., Morgan, P. T., Malison, R. T., Hart, C. L., Edgar, C., Walker, M., et al. (2008). Cocaine users differ from normals on cognitive tasks which show poorer performance during drug abstinence. The American Journal of Drug and Alcohol Abuse, 34(1), 109121.

Peña-Casanova, J. (1991). Programa Integrado de Exploración Neuropsicológica "Test Barcelona". Normalidad, Semiología y patología neuropsicológicas. Barcelona: Masson.

Peña-Casanova, J., Gramunt Fombuena, N., \& Gich Fullá, J. (2006). Test neuropsicológicos. Fundamentos para una neuropsicología clínica basada en evidencias. Barcelona: Masson.

Perianez, J. A., Rios-Lago, M., Rodriguez-Sanchez, J. M., Adrover-Roig, D., Sanchez-Cubillo, I., Crespo-Facorro, B., et al. (2007). Trail Making Test in traumatic brain injury, schizophrenia, and normal ageing: sample comparisons and normative data. Archives of Clinical Neuropsychology, 22(4), 433-447.

Ramos-Cejudo, J., \& Iruarrizaga Díez, I. (2009). Correlatos Neuropsicológicos y Emocionales implicados en el Consumo de Cocaína: Una Revisión Teórica a los Nuevos Hallazgos. Intervención Psicosocial, 18(3), 245-253. 
Reitan, R. M., \& Wolfson, D. (1985). The Halstead-Reitan Neuropsychological Battery. Theory and clinical interpretation. Tucson, AZ: Neuropsychology Press.

Roberts, M. E., Tchanturia, K., Stahl, D., Southgate, L., \& Treasure, J. (2007). A systematic review and meta-analysis of set-shifting ability in eating disorders. Psychological Medicine, 37(8), 1075-1084.

Rosselli, M., Ardila, A., Lubomski, M., Murray, S., \& King, K. (2001). Personality profile and neuropsychological test performance in chronic cocaine-abusers. The International Journal of Neuroscience, 110(1-2), 55-72.

Sánchez-Hervás, E., Secades-Villa, R, Santonja Gómez, J. F. Zacarés Romaguera, F., \& García-Rodríguez, O. (2009). Addictive severity in cocaine addicts measured with the EuropASI: differences between composite scores and severity ratings. The Amercian Journal on Addictions, 18(5), 375-378.

Sánchez Hervás, E., Tomas Gradoli, V., \& Morales Gallus, E. (2000). Psychopathologic evaluation of cocaine-dependent patients. Atención Primaria, 26(5), 319-322.

Satel, S. L., Kosten, T. R., Schuckit, M. A., \& Fischman, M. W. (1993). Should protracted withdrawal from drugs be included in DSM-IV? American Journal of Psychiatry, 150(5), 695-704.

Schrimsher, G. W., \& Parker, J. D. (2008). Changes in Cognitive Function During Substance Use Disorder Treatment. Journal of Psychopathology and Behavioral Assessment, 30(2), 146-153.

Selzer, M. L. (1971). The Michigan alcoholism screening test: the quest for a new diagnostic instrument. The American Journal of Psychiatry, 127(12), 1653-1658.

Severtson, S. G., von Thomsen, S., Hedden, S. L., \& Latimer, W. (2010). The association between executive functioning and motivation to enter treatment among regular users of heroin and/or cocaine in Baltimore, MD. Addictive Behaviors, 35(7), 717-720.
Shields, A. L., Howell, R. T., Potter, J. S., \& Weiss, R. D. (2007). The Michigan Alcoholism Screening Test and its shortened form: a metaanalytic inquiry into score reliability. Substance Use and Misuse, 42(11), 1783-1800.

Sociedad Española de Toxicomanías. (2011). Neurociencia y adicción. Madrid: Delegación del Gobierno para el Plan Nacional sobre Drogas.

Terán, A., Casete, L., \& Climent, B. (2008). Cocaína. Guías Clínicas basada en la Evidencia Científica. Valencia: Socidrogalcohol.

Tombaugh, T. N. (2004). Trail Making Test A and B: normative data stratified by age and education. Archives of Clinical Neuropsychology, 19(2), 203-214.

Vázquez Valverde, C., \& López Luengo, B. (2003). Un estudio sistemático de la evolución de los déficit cognitivos en una muestra de pacientes con esquizofrenia. Revista de psiquiatría de la Facultad de Medicina de Barcelona, 30(3), 129-136.

Verdejo-García, A., \& Bechara, A. (2009). Neuropsicología y drogodependencias: evaluación, impacto clínico y aplicaciones para la rehabilitación. In M. Pérez García (Ed.), Manual de neuropsicología clínica (pp. 179-208). Madrid: Pirámide.

Verdejo-García, A., López-Torrecillas, F., Gimenez, C. O., \& Pérez-García, M. (2004). Clinical implications and methodological challenges in the study of the neuropsychological correlates of cannabis, stimulant, and opioid abuse. Neuropsychology Review, 14(1), 1-41.

Verdejo, A., Orozco-Gimenez, C., Meersmans Sánchez-Jofre, M., Aguilar de Arcos, F., \& Pérez-García, M. (2004). The impact exerted by the severity of recreational drug abuse on the different components of the executive function. Revista de Neurología, 38(12), 1109-1116.

(Articulo recibido: 27-4-2010, revisado: 5-11-2011, aceptado: 12-11-2011) 